\title{
Implementation of a non-oscillatory and conservative scheme into magnetohydrodynamic equations
}

\author{
Shin Tanaka ${ }^{1 *}$, Takayuki Umeda ${ }^{1}$, Yosuke Matsumoto ${ }^{1,2}$, Takahiro Miyoshi ${ }^{3}$, and Tatsuki Ogino ${ }^{1}$ \\ ${ }^{1}$ Solar-Terrestrial Environment Laboratory, Nagoya University, Nagoya, Aichi 464-8601, Japan \\ ${ }^{2}$ Graduate School of Environmental Studies, Nagoya University, Nagoya, Aichi 464-8601, Japan \\ ${ }^{3}$ Department of Physical Science, Graduate School of Science, Hiroshima University, \\ Higashi-Hiroshima, Hiroshima 739-8526, Japan
}

(Received December 21, 2007; Revised January 20, 2009; Accepted February 12, 2009; Online published August 31, 2009)

\begin{abstract}
We present a magnetohydrodynamic (MHD) simulation technique with a new non-oscillatory and conservative interpolation scheme. Several high-resolution and stable numerical schemes have recently been proposed for solving the MHD equations. To apply the CIP scheme to the hydrodynamic equations, we need to add a certain diffusion term to suppress numerical oscillations at discontinuities. Although the TVD schemes can automatically avoid numerical oscillations, they are not appropriate for profiles with a local maximum or minimum, such as waves. To deal with the above problems, we implement a new non-oscillatory and conservative interpolation scheme in MHD simulations. Several numerical tests are carried out in order to compare our scheme with other recent high-resolution schemes. The numerical tests suggest that the present scheme can follow long-term evolution of both Alfvén waves and compressive shocks. The present scheme has been used for a numerical modeling of Alfvén waves in the solar wind, in which sinusoidal Alfvén waves decay into compressive sound waves that steepen into shocks.
\end{abstract}

Key words: MHD, numerical scheme, hyperbolic equation, conservative scheme.

\section{Introduction}

Magnetohydrodynamic (MHD) simulation techniques are widely used to study various global and macroscopic phenomena in plasmas. It is important to develop accurate, efficient, stable, and robust numerical schemes for the MHD simulations. In the laboratory, astrophysical, and space plasmas often appear as high-speed flows that form shocks and discontinuities. Standard numerical schemes, such as the Lax-Wendroff scheme, generate spurious oscillations in a steep profile and therefore need additional numerical diffusion terms, such as artificial viscosity, to capture shocks and discontinuities.

Several Roe-type Riemann solvers have been developed to obtain more accurate numerical solutions of the MHD equations (Roe, 1981; Brio and Wu, 1988; Dai and Woodward, 1994; Ryu and Jones, 1995). Although those Riemann solvers are much more complicated than simple finite difference methods, they are widely used because of their stability. Miyoshi and Kusano (2005) recently developed the Harten-Lax-van Leer Discontinuities (HLLD) approximate Riemann solver for the MHD equations. The HLLD Riemann solver has the advantages of positivity preserving and accuracy in comparison with the linearized Riemann solver while keeping the latter's robustness.

The high-resolution upwind schemes based on the To-

*Now at Rigaku Corporation, Japan.

Copyright (c) The Society of Geomagnetism and Earth, Planetary and Space Sciences (SGEPSS); The Seismological Society of Japan; The Volcanological Society of Japan; The Geodetic Society of Japan; The Japanese Society for Planetary Sciences; TERRAPUB tal Variation Diminishing (TVD) scheme are also implemented into the MHD equations (Tanaka, 1994; Fukuda and Hanawa, 1999). The TVD schemes have the advantages of being able to capture shocks and discontinuities more accurately without additional numerical diffusion terms. However, the TVD schemes are especially not appropriate for the profile with extrema, i.e., local maxima and minima, such as waves, as they tend to make profiles flat-top and flat-bottom to maintain monotonicity.

Another high-resolution scheme called the CIP-MOCCT scheme (Kudoh and Shibata, 1997) for solving the MHD equations, which is based on the Constrained Interpolation Profile (CIP) scheme (Yabe et al., 2001) and the Method Of Characteristics-Constrained Transport (MOCCT) scheme (Hawley and Stone, 1995). The CIP scheme has been developed for solving hyperbolic equations. The MOCCT scheme solves the induction equation and magnetic stress in the MHD equation, maintaining the divergence-free condition of the magnetic field i.e., $\nabla \cdot \mathbf{B}=0$. However, there are several problems associated with the CIP-MOCCT scheme. It needs to add a certain diffusion term to suppress numerical oscillation, and it also needs a larger computer memory than other high-resolution schemes for the MHD equations in order to store partial derivatives or integrals of a profile.

A new interpolation scheme has recently been developed for the Vlasov equation (Umeda, 2008). This scheme is a non-oscillatory, positivity preserving, and conservative numerical interpolation scheme for solving the linear advection equation. Recent numerical tests have demonstrated that the non-oscillatory scheme has advantages over the CIP 
and TVD schemes in Vlasov simulations (Umeda et al., 2006; Umeda, 2008).

We report here our attempt to implement the nonoscillatory scheme to develop a new MHD simulation code. The original non-oscillatory scheme is designed for the linear advection equation in which an advection velocity is constant (Umeda, 2008). Thus, we first extend the non-oscillatory scheme to the general advection equation. The extended non-oscillatory scheme is then applied to the MHD equations in a manner quite similar to the CIPMOCCT scheme, whereas we use the conservative forms of the MHD equations. To solve the induction equation and magnetic stress, we adopt the MOCCT scheme. In the MOCCT scheme, the van Leer interpolation (van Leer, 1974,1977 ) has been generally used to interpolate the magnetic field and velocity to suppress numerical oscillations at discontinuities. As an alternative to the van Leer interpolation, we also use the non-oscillatory interpolation and examine their differences. We compare our schemes with other recent numerical schemes by test simulations.

The organization of the paper is as follows. We describe our new scheme for MHD equations in Section 2. We also briefly introduce the non-oscillatory scheme and MOCCT scheme in this section. In Section 3, we show results of one-dimensional test runs. A summary and discussion of this paper are given in Section 4.

\section{Numerical Method}

\subsection{Basic equations}

We assume a 1.5-dimensional system along a uniform ambient magnetic field in which all quantities depend only on $x$. We solve the following set of the ideal MHD equations in a conservative form

$$
\begin{gathered}
\frac{\partial \rho}{\partial t}+\frac{\partial}{\partial x}\left(\rho v_{x}\right)=0 \\
\frac{\partial M_{x}}{\partial t}+\frac{\partial}{\partial x}\left(M_{x} v_{x}\right)=-\frac{\partial}{\partial x}\left(p+\frac{B_{y}^{2}}{2 \mu_{0}}\right), \\
\frac{\partial M_{y}}{\partial t}+\frac{\partial}{\partial x}\left(M_{y} v_{x}\right)=\frac{B_{x}}{\mu_{0}} \frac{\partial B_{y}}{\partial x}, \\
\frac{\partial e}{\partial t}+\frac{\partial}{\partial x}\left\{\left(e+p+\frac{B_{y}^{2}}{2 \mu_{0}}\right) v_{x}\right\}=\frac{B_{x}}{\mu_{0}} \frac{\partial}{\partial x}\left(v_{y} B_{y}\right), \\
\frac{\partial B_{y}}{\partial t}=\frac{\partial E_{z}}{\partial x}, \\
E_{z}=-v_{x} B_{y}+v_{y} B_{x},
\end{gathered}
$$

where

$$
e=\rho \frac{v_{x}^{2}+v_{y}^{2}}{2}+\frac{p}{\gamma-1}+\frac{B_{y}^{2}}{2 \mu_{0}},
$$

represents the total energy, and $\rho, p, v_{x}, v_{y}, B_{x}, B_{y}, E_{z}$, $\mu_{0}$, and $\gamma$ represent the density, pressure, velocity components in the $x$ and $y$ directions, magnetic field components in the $x$ and $y$ directions, electric field component in the $z$ direction, magnetic permeability in vacuum, and the ratio of specific heats, respectively. Here the momentum components in the $x$ and $y$ directions are defined as $M_{x} \equiv \rho v_{x}$ and $M_{y} \equiv \rho v_{y}$, respectively. The magnetic field $B_{x}$ is constant in time and space due to the $\nabla \cdot \mathbf{B}=0$ constraint. We ne-

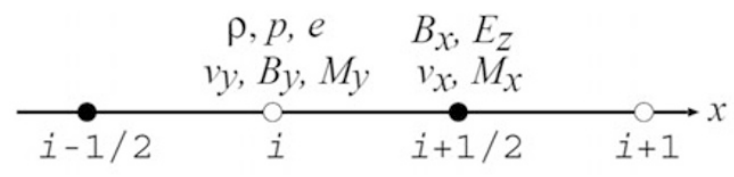

Fig. 1. Grid assignment of the present scheme.

glected the $z$ component of the velocity and magnetic field in basic equations for simplicity.

We define full-integer grids at $i \Delta x(i=1,2,3, \cdots, N)$ and half-integer grids at $(i+1 / 2) \Delta x$. The quantities $\rho, p$, $e, v_{y}, M_{y}$, and $B_{y}$ are defined at the full-integer grids, while $v_{x}, M_{x}, B_{x}$, and $E_{z}$ are defined at the half-integer grids, as shown in Fig. 1.

To apply the non-oscillatory and MOCCT schemes, we define the left-hand side of Eqs. (1)-(4) as "advection phase", and the right-hand side of Eqs. (2)-(4) as "nonadvection phase". The advection phase is solved with the non-oscillatory scheme, while the non-advection phase is separately solved with other schemes.

\subsection{Non-oscillatory and conservative scheme}

Here we briefly review the non-oscillatory scheme and extend it for a general advection equation. We consider the following generalized one-dimensional advection equation

$$
\frac{\partial f}{\partial t}+\frac{\partial}{\partial x}(v g)=0
$$

where $f, g$, and $v$ are functions of $x$ and $t$. A solution of Eq. (8) in the conservative form is written as

$$
f_{i}^{t+\Delta t}=f_{i}^{t}+U_{i-\frac{1}{2}}\left(v_{i-\frac{1}{2}}\right)-U_{i+\frac{1}{2}}\left(v_{i+\frac{1}{2}}\right),
$$

where $U_{i+\frac{1}{2}}$ represents a numerical flux at $x_{i+\frac{1}{2}}$. The numerical flux is a function of the CFL number $v_{i+\frac{1}{2}}=-v_{i+\frac{1}{2}} \frac{\Delta t}{\Delta x}$, where $\Delta t$ and $\Delta x$ are a constant time step and a constant grid spacing, respectively. We assume that the advection velocity is constant in short spatial and time scales (i.e., $\Delta x$ and $\Delta t$ ) so that we can apply the scheme to Eq. (9).

The numerical flux of the third-order upwind-biased Lagrange polynomial interpolation is easily obtained by the following constraints, $U_{i+\frac{1}{2}}(0)=0, U_{i+\frac{1}{2}}(1)=f_{i}$, $U_{i+\frac{1}{2}}(2)=f_{i-1}$, and $U_{i+\frac{1}{2}}(-1)=-f_{i+1}$. In the nonoscillatory scheme (Umeda, 2008), a flux limiter/slope corrector is introduced into the third-order upwind-biased Lagrange polynomial in order to suppress the generation new extrema, i.e., local maximum or minimum. Thus, the numerical flux of the non-oscillatory scheme is given as

$$
\begin{aligned}
& U_{i+\frac{1}{2}}\left(v_{i+\frac{1}{2}}\right)=v_{i+\frac{1}{2}} g_{i}+v_{i+\frac{1}{2}}\left(1-v_{i+\frac{1}{2}}\right)\left(2-v_{i+\frac{1}{2}}\right) \frac{L_{i}^{(+)}}{6} \\
& +v_{i+\frac{1}{2}}\left(1-v_{i+\frac{1}{2}}\right)\left(1+v_{i+\frac{1}{2}}\right) \frac{L_{i}^{(-)}}{6} \\
& \left(1 \geq v_{i+\frac{1}{2}} \geq 0\right) \text { if } v_{i+\frac{1}{2}} \geq 0 \text {, } \\
& U_{i+\frac{1}{2}}\left(v_{i+\frac{1}{2}}\right)=v_{i+\frac{1}{2}} g_{i+1}-v_{i+\frac{1}{2}}\left(1+v_{i+\frac{1}{2}}\right)\left(2+v_{i+\frac{1}{2}}\right) \frac{L_{i+1}^{(-)}}{6} \\
& -v_{i+\frac{1}{2}}\left(1-v_{i+\frac{1}{2}}\right)\left(1+v_{i+\frac{1}{2}}\right) \frac{L_{i+1}^{(+)}}{6}
\end{aligned}
$$




$$
\left(0 \geq v_{i+\frac{1}{2}} \geq-1\right) \text { if } v_{i+\frac{1}{2}}<0 \text {. }
$$

where

$$
\begin{aligned}
L_{i}^{(+)} & = \begin{cases}\min \left[2\left(g_{i}-g_{\min }\right),\left(g_{i+1}-g_{i}\right)\right] & \text { if } g_{i+1} \geq g_{i} \\
\max \left[2\left(g_{i}-g_{\max }\right),\left(g_{i+1}-g_{i}\right)\right] & \text { if } g_{i+1}<g_{i}\end{cases} \\
L_{i}^{(-)} & = \begin{cases}\min \left[2\left(g_{\max }-g_{i}\right),\left(g_{i}-g_{i-1}\right)\right] & \text { if } g_{i} \geq g_{i-1} \\
\max \left[2\left(g_{\min }-g_{i}\right),\left(g_{i}-g_{i-1}\right)\right] & \text { if } g_{i}<g_{i-1}\end{cases}
\end{aligned}
$$
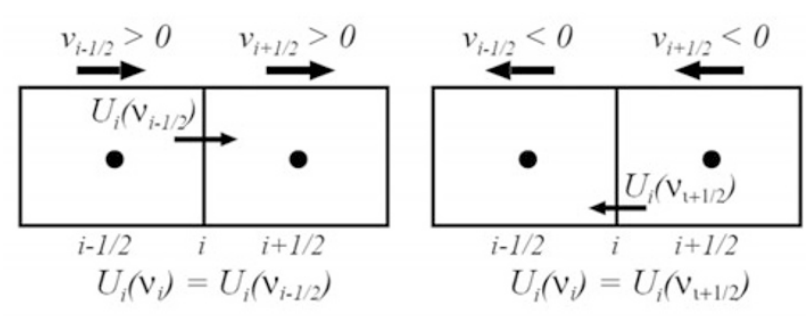

are a non-oscillatory flux limiter with

$$
\begin{aligned}
g_{\max } & =\max \left[g_{\max 1}, g_{\max 2}\right], \\
g_{\min } & =\min \left[g_{\min 1}, g_{\min 2}\right],
\end{aligned}
$$

and

$g_{\max 1}=\max \left[\max \left[g_{i-1}, g_{i}\right], \min \left[2 g_{i-1}-g_{i-2}, 2 g_{i}-g_{i+1}\right]\right]$, $g_{\max 2}=\max \left[\max \left[g_{i+1}, g_{i}\right], \min \left[2 g_{i+1}-g_{i+2}, 2 g_{i}-g_{i-1}\right]\right]$, $g_{\min 1}=\min \left[\min \left[g_{i-1}, g_{i}\right], \max \left[2 g_{i-1}-g_{i-2}, 2 g_{i}-g_{i+1}\right]\right]$, $g_{\min 2}=\min \left[\min \left[g_{i+1}, g_{i}\right], \max \left[2 g_{i+1}-g_{i+2}, 2 g_{i}-g_{i-1}\right]\right]$.

Note that the minimum principle switches to

$$
g_{\min }=\max \left[0, \min \left[g_{\min 1}, g_{\min 2}\right]\right],
$$

if we need to preserve positivity.

The basic concept of the non-oscillatory scheme is to capture the shape of a profile by detecting a local maximum $g_{\max }$ or minimum $g_{\min }$ of a piecewise interpolant. When a profile is continuous so that can be interpolated by the third-order polynomial without the non-oscillatory scheme, it switches to the Lagrange polynomial interpolation. When a profile has a discontinuity, the gradient (slope) of the interpolant is corrected so that the piecewise interpolant does not generate inadequate extrema.

In this paper we define a conservation equation (9) as a following non-oscillatory operator,

$$
\begin{aligned}
f_{i}^{t+\Delta t} & =f_{i}^{t}+U_{i-\frac{1}{2}}\left(v_{i-\frac{1}{2}}\right)-U_{i+\frac{1}{2}}\left(v_{i+\frac{1}{2}}\right) \\
& \equiv \mathrm{NO}\left[f_{i}^{t}, g_{i}^{t}, v_{i+\frac{1}{2}} \Delta t, v_{i-\frac{1}{2}} \Delta t\right] .
\end{aligned}
$$

Note that numerical fluxes $U_{i \pm \frac{1}{2}}$ are computed using $g_{i}$ and $v_{i \pm \frac{1}{2}}$. When a profile is defined at a half-integer grid such as $g_{i+\frac{1}{2}}$, we need advection velocities at a full-integer grid $v_{i}$ and $^{2} v_{i+1}$ to compute numerical fluxes at a full-integer grid $U_{i}$ and $U_{i+1}$ in the operator Eq. (10). Since an advection velocity is not defined at a full-integer grid, we evaluate $U_{i}\left(v_{i}\right)$ according to a sign of $v_{i \pm \frac{1}{2}}$ as follows (e.g., Hirsch, 1990),

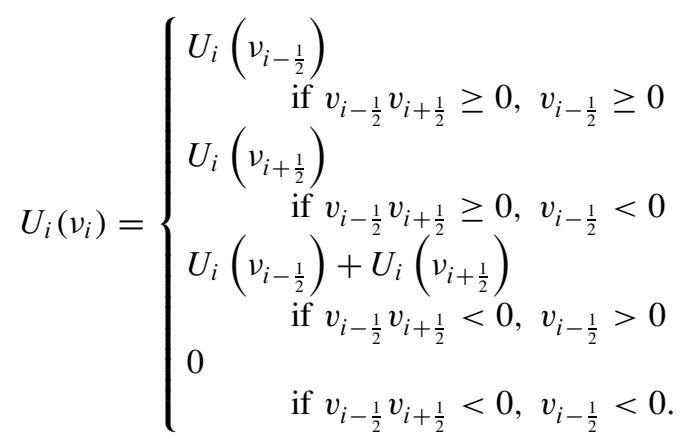

Figure 2 shows the above four patterns of diagrams for computation of numerical flux $U_{i}\left(v_{i}\right)$ with Eq. (11).
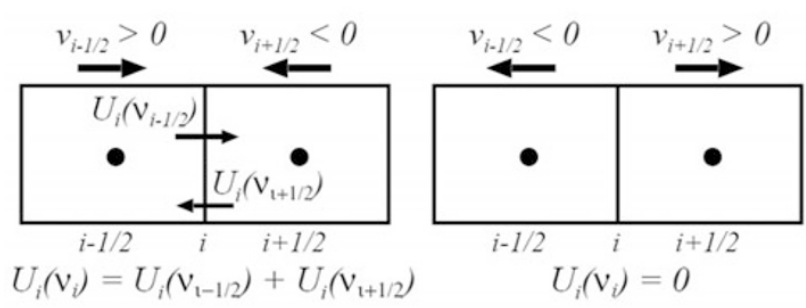

Fig. 2. Diagram of a computation of a numerical flux at a full-integer grid $U_{i}\left(v_{i}\right)$ (see Eq. (11)).

\subsection{The method of characteristics}

The MOC provides the time-advanced transverse velocity $v_{y, i+\frac{1}{2}}$ and magnetic field $B_{y, i+\frac{1}{2}}$ at $t+\frac{\Delta t}{2}$. Assuming that the fluid is incompressible in which $\rho$ and $v_{x}$ are constant, Eqs. (3), (5), and (6) can be reduced to the following Alfvén characteristic equations

$$
\begin{aligned}
& \left(\frac{\partial}{\partial t}+C^{+} \frac{\partial}{\partial x}\right) A^{-}=0 \\
& \left(\frac{\partial}{\partial t}+C^{-} \frac{\partial}{\partial x}\right) A^{+}=0
\end{aligned}
$$

where

$$
\begin{aligned}
& A^{ \pm}=v_{y} \pm \frac{B_{y}}{\sqrt{\mu_{0} \rho}}, \\
& C^{ \pm}=v_{x} \pm \frac{B_{x}}{\sqrt{\mu_{0} \rho}} .
\end{aligned}
$$

These equations mean that the quantities $A^{\mp}$ are conserved along the characteristics $C^{ \pm}$. Thus, $v_{y, i+\frac{1}{2}}^{t+\frac{\Delta t}{2}}$ and $B_{y, i+\frac{1}{2}}^{t+\frac{\Delta t}{2}}$ are given by

$$
\begin{aligned}
v_{y, i+\frac{1}{2}}^{t+\frac{\Delta t}{2}} & =\frac{1}{2}\left(A_{i+\frac{1}{2}}^{+, t+\frac{\Delta t}{2}}+A_{i+\frac{1}{2}}^{-, t+\frac{\Delta t}{2}}\right), \\
B_{y, i+\frac{1}{2}}^{t+\frac{\Delta t}{2}} & =\frac{\sqrt{\mu_{0} \rho}}{2}\left(A_{i+\frac{1}{2}}^{+, t+\frac{\Delta t}{2}}-A_{i+\frac{1}{2}}^{-, t+\frac{\Delta t}{2}}\right) .
\end{aligned}
$$

We compute $A_{i+\frac{1}{2}}^{+, t+\frac{\Delta t}{2}}$ solving the advection equation (13) with the following simple interpolation,

$$
A_{i+\frac{1}{2}}^{+, t+\frac{\Delta t}{2}}=\left\{\begin{array}{r}
A_{i}^{+, t}+\frac{1}{2}\left(\Delta x-C_{i+\frac{1}{2}}^{-} \Delta t\right)\left(\frac{\Delta A}{\Delta x}\right)_{i} \\
\text { if } C_{i+\frac{1}{2}}^{-}>0 \\
A_{i+1}^{+, t}-\frac{1}{2}\left(\Delta x+C_{i+\frac{1}{2}}^{-} \Delta t\right)\left(\frac{\Delta A}{\Delta x}\right)_{i+1} \\
\text { if } C_{i+\frac{1}{2}}^{-}<0
\end{array}\right.
$$

where

$$
A_{i}^{+, t}=v_{y, i}^{t}+\frac{B_{y, i}^{t}}{\sqrt{\mu_{0} \rho_{i}^{t}}},
$$




$$
C_{i+\frac{1}{2}}^{-}=v_{x, i+\frac{1}{2}}-\frac{B_{x}}{\sqrt{\mu_{0} \frac{\rho_{i}^{t}+\rho_{i+1}^{t}}{2}}},
$$

with the van Leer limiter (van Leer, 1974, 1977)

$$
\left(\frac{\Delta A}{\Delta x}\right)_{i}=\left\{\begin{array}{c}
\frac{2}{\Delta x} \frac{\left(A_{i+1}^{+, t}-A_{i}^{+, t}\right)\left(A_{i}^{+, t}-A_{i-1}^{+, t}\right)}{\left(A_{i+1}^{+, t}-A_{i}^{+, t}\right)+\left(A_{i}^{+, t}-A_{i-1}^{+, t}\right)} \\
\text { if }\left(A_{i+1}^{+, t}-A_{i}^{+, t}\right)\left(A_{i}^{+, t}-A_{i-1}^{+, t}\right)>0 \\
0 \quad \text { if }\left(A_{i+1}^{+, t}-A_{i}^{+, t}\right)\left(A_{i}^{+, t}-A_{i-1}^{+, t}\right)<0 .
\end{array}\right.
$$

We also compute $A_{i+\frac{1}{2}}^{-, t+\frac{\Delta t}{2}}$ by solving Eq. (12) for the characteristic $C^{+}$in the same way.

The van Leer interpolation described above has been used for the MOCCT (Hawley and Stone, 1995; Kudoh and Shibata, 1997). We can alternatively use the non-oscillatory interpolation to compute $A_{i+\frac{1}{2}}^{+, t+\frac{\Delta t}{2}}$, such as

$$
\begin{aligned}
A_{i+\frac{1}{2}}^{+, t+\frac{\Delta t}{2}}= & \mathrm{NO}\left[A_{i+1}^{+, t+\frac{\Delta t}{2}}, A_{i+1}^{+, t+\frac{\Delta t}{2}}, \frac{1}{2}\left(\Delta x-C_{i+\frac{1}{2}}^{-} \Delta t\right)\right. \\
& \left.\frac{1}{2}\left(\Delta x-C_{i+\frac{1}{2}}^{-} \Delta t\right)\right] .
\end{aligned}
$$

The difference between the van Leer interpolation Eq. (16) and the non-oscillatory interpolation Eq. (18) will be examined by numerical tests in Section 3 .

In this paper we define the following MOC operator for simplicity

$$
\begin{aligned}
\left(v_{y, i+\frac{1}{2}}^{t+\frac{\Delta t}{2}}, B_{y, i+\frac{1}{2}}^{t+\frac{\Delta t}{2}}\right)= & \operatorname{MOC}\left[v_{y, i}^{t}, v_{y, i+1}^{t}, B_{y, i}^{t}, B_{y, i+1}^{t},\right. \\
& \left.\rho_{i}^{t}, \rho_{i+1}^{t}, v_{x, i+\frac{1}{2}}\right] .
\end{aligned}
$$

\subsection{Time step chart}

The physical quantities in Eqs. (1)-(5) are advanced in time based on the sequences shown in Fig. 3. The time step chart of the present scheme is similar to that of the CIPMOCCT scheme (Kudoh and Shibata, 1997).

1) We solve the non-advection phase in Eqs. (3) and (4), i.e., the terms associated with the magnetic stress. We compute the time-advanced transverse velocity $\tilde{v}_{y, i+\frac{1}{2}}$ and magnetic field $\tilde{B}_{y, i+\frac{1}{2}}$ with the MOC.

$$
\begin{aligned}
\left(\tilde{v}_{y, i+\frac{1}{2}}, \tilde{B}_{y, i+\frac{1}{2}}\right)= & \operatorname{MOC}\left[v_{y, i}^{t}, v_{y, i+1}^{t}, B_{y, i}^{t}, B_{y, i+1}^{t},\right. \\
& \left.\rho_{i}^{t}, \rho_{i+1}^{t}, 0\right]
\end{aligned}
$$

It should be noted that $v_{x}$ is set to be 0 in the MOC operator, which means that the characteristic velocity does not include the advection velocity. This is because the advection phase of the momentum equation is separately solved with the non-oscillatory scheme. The time-advanced values of the transverse momentum $M_{y, i}^{*}$ and the total energy $e_{i}^{*}$ associated with the non-advection phase are then given by

$$
M_{y, i}^{*}=M_{y, i}^{t}+\frac{\Delta t}{\Delta x} \frac{B_{x}}{\mu_{0}}\left(\tilde{B}_{y, i+\frac{1}{2}}-\tilde{B}_{y, i-\frac{1}{2}}\right)
$$

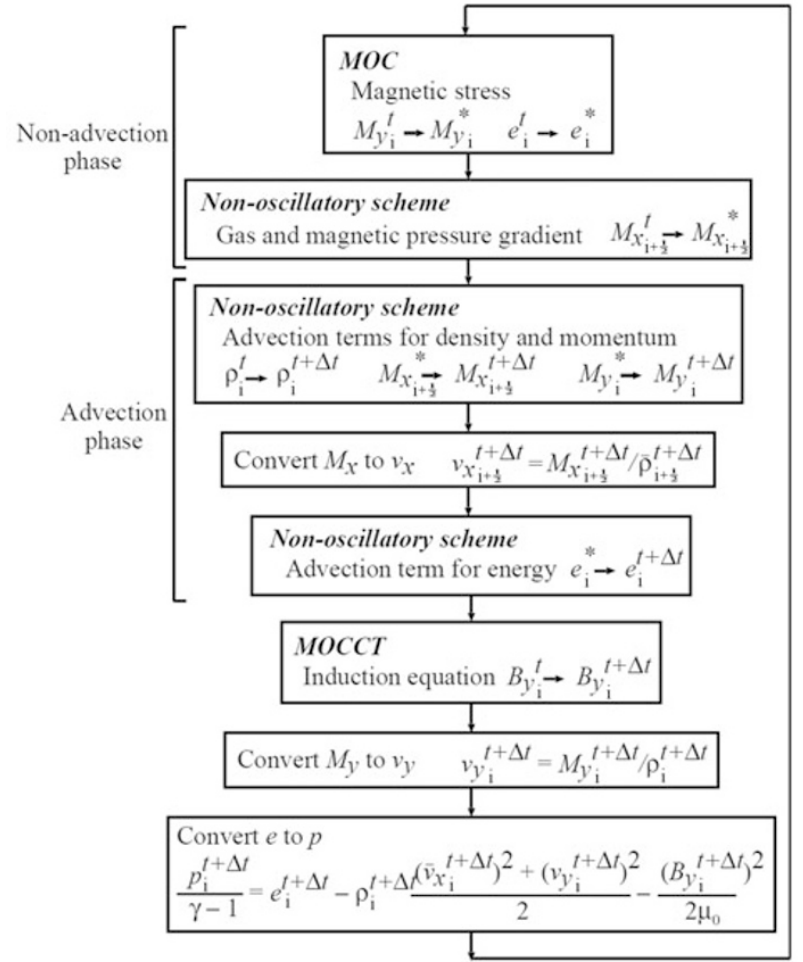

Fig. 3. Time step chart of the present scheme. Note that $\bar{\rho}$ and $\bar{v}_{x}$ are assigned at half-integer and full-integer grids, respectively, in contrast to Fig. 1.

$$
e_{i}^{*}=e_{i}^{t}+\frac{\Delta t}{\Delta x} \frac{B_{x}}{\mu_{0}}\left(\tilde{B}_{y, i+\frac{1}{2}} \tilde{v}_{y, i+\frac{1}{2}}-\tilde{B}_{y, i-\frac{1}{2}} \tilde{v}_{y, i-\frac{1}{2}}\right) .
$$

Note that $M_{y}$ and $e$ are advanced in a conservative form.

2) The non-advection phase of Eq. (2), i.e., the gas and magnetic pressure term, is solved with the nonoscillatory interpolation. We estimate the gas and magnetic pressure term at half-integer grids. The pressure gradient at the half-integer grids is then computed by directly differentiating them with the nonoscillatory operator as follows:

$$
\begin{gathered}
\left\{\frac{\partial}{\partial x}\left(p+\frac{B_{y}^{2}}{2 \mu_{0}}\right)\right\}_{i+\frac{1}{2}}=\frac{\partial}{\partial x} \mathrm{NO}\left[\left(p+\frac{B_{y}^{2}}{2 \mu_{0}}\right)_{i+1},\right. \\
\left.\left(p+\frac{B_{y}^{2}}{2 \mu_{0}}\right)_{i+1}, \frac{\Delta x}{2}, \frac{\Delta x}{2}\right] .
\end{gathered}
$$

The time-advanced value of the longitudinal momentum $M_{x, i+\frac{1}{2}}^{*}$ associated with the non-advection phase is given by

$$
M_{x, i+\frac{1}{2}}^{*}=M_{x, i+\frac{1}{2}}^{t}-\Delta t\left\{\frac{\partial}{\partial x}\left(p+\frac{B_{y}^{2}}{2 \mu_{0}}\right)\right\}_{i+\frac{1}{2}} .
$$

Here we applied the Euler time-integration scheme in which $M_{x}$ is advanced in a conservative form. 
3) We solve the advection phase of Eqs. (1), (2), and (3) with the non-oscillatory scheme.

$$
\begin{aligned}
\rho_{i}^{t+\Delta t} & =\mathrm{NO}\left[\rho_{i}^{t}, \rho_{i}^{t}, v_{x, i+\frac{1}{2}}^{t} \Delta t, v_{x, i-\frac{1}{2}}^{t} \Delta t\right], \\
M_{x, i+\frac{1}{2}}^{t+\Delta t} & =\mathrm{NO}\left[M_{x, i+\frac{1}{2}}^{*}, M_{x, i+\frac{1}{2}}^{*}, v_{x, i+1}^{t} \Delta t, v_{x, i}^{t} \Delta t\right] \\
M_{y, i}^{t+\Delta t} & =\mathrm{NO}\left[M_{y, i}^{*}, M_{y, i}^{*}, v_{x, i+\frac{1}{2}}^{t} \Delta t, v_{x, i-\frac{1}{2}}^{t} \Delta t\right] .
\end{aligned}
$$

Note that since $v_{x}$ at full-integer grids in Eq. (26) are unknown, numerical fluxes at full-integer grids are given by Eq. (11).

4) We update the velocity component $v_{x}^{t+\Delta t}$ with $\rho^{t+\Delta t}$ and $M_{x}^{t+\Delta t}$. Since $\rho$ is defined at full-integer grids, $\bar{\rho}_{i+\frac{1}{2}}^{t+\Delta t}$ is computed with the non-oscillatory interpolation, i.e.,

$$
\bar{\rho}_{i+\frac{1}{2}}^{t+\Delta t}=\mathrm{NO}\left[\rho_{i+1}^{t+\Delta t}, \rho_{i+1}^{t+\Delta t}, \frac{\Delta x}{2}, \frac{\Delta x}{2}\right] .
$$

We then obtain $v_{x}$ at $t+\Delta t$ as

$$
v_{x, i+\frac{1}{2}}^{t+\Delta t}=\frac{M_{x, i+\frac{1}{2}}^{t+\Delta t}}{\bar{\rho}_{i+\frac{1}{2}}^{t+\Delta t}}
$$

5) We solve the advection phase of Eq. (4) with the nonoscillatory scheme to obtain $e^{t+\Delta t}$.

$$
\begin{aligned}
e_{i}^{t+\Delta t}= & \mathrm{NO}\left[e_{i}^{*}, e_{i}^{*}+p_{i}^{t}+\frac{\left(B_{y, i+\frac{1}{2}}^{t}\right)^{2}}{2 \mu_{0}},\right. \\
& \left.v_{x, i+\frac{1}{2}}^{t+\Delta t} \Delta t, v_{x, i-\frac{1}{2}}^{t+\Delta t} \Delta t\right]
\end{aligned}
$$

Note that we use values of $v_{x}$ at $t+\Delta t$ as the advection velocity. This is to obtain a more desirable result of the MHD shock tube problem shown in Section 3.

6) We solve the induction equation (5) with the MOCCT scheme. First, we compute the time-advanced transverse velocity $\bar{v}_{y, i+\frac{1}{2}}$ and magnetic field $\bar{B}_{y, i+\frac{1}{2}}$ with the MOC,

$$
\begin{aligned}
\left(\bar{v}_{y, i+\frac{1}{2}}, \bar{B}_{y, i+\frac{1}{2}}\right)= & \operatorname{MOC}\left[v_{y, i}^{t}, v_{y, i+1}^{t}, B_{y, i}^{t}, B_{y, i+1}^{t}\right. \\
& \left.\rho_{i}^{t}, \rho_{i+1}^{t}, v_{x, i+\frac{1}{2}}^{t+\Delta t}\right]
\end{aligned}
$$

We next compute electro-motive force $\bar{\varepsilon}_{i+\frac{1}{2}}$, which is necessary for the CT scheme (Hawley and Stone, 1995).

$$
\bar{\varepsilon}_{i+\frac{1}{2}}=v_{x, i+\frac{1}{2}}^{t+\Delta t} \bar{B}_{y, i+\frac{1}{2}}-\bar{v}_{y, i+\frac{1}{2}} B_{x} .
$$

$B_{y}$ is then advanced by discretizing Eq. (5) as follows

$$
B_{y, i+\frac{1}{2}}^{t+\Delta t}=B_{y, i+\frac{1}{2}}^{t}-\frac{\Delta t}{\Delta x}\left(\bar{\varepsilon}_{i+\frac{1}{2}}-\bar{\varepsilon}_{i-\frac{1}{2}}\right)
$$

7) Finally, we compute $v_{y}^{t+\Delta t}$ and $p^{t+\Delta t}$ as follows:

$$
\begin{aligned}
v_{y, i}^{t+\Delta t}= & \frac{M_{y, i}^{t+\Delta t}}{\rho_{i}^{t+\Delta t}}, \\
\frac{p_{i}^{t+\Delta t}}{\gamma-1}= & e_{i}^{t+\Delta t}-\rho_{i}^{t+\Delta t} \frac{\left(\bar{v}_{x, i}^{t+\Delta t}\right)^{2}+\left(v_{y, i}^{t+\Delta t}\right)^{2}}{2} \\
& -\frac{\left(B_{y, i}^{t+\Delta t}\right)^{2}}{2 \mu_{0}} .
\end{aligned}
$$

Here we use Eq. (7) to convert energy to pressure. Note that $\bar{v}_{x, i}^{t+\Delta t}$ is computed with the non-oscillatory operator, i.e.,

$$
\bar{v}_{x, i}^{t+\Delta t}=\mathrm{NO}\left[v_{x, i+\frac{1}{2}}^{t+\Delta t}, v_{x, i+\frac{1}{2}}^{t+\Delta t}, \frac{\Delta x}{2}, \frac{\Delta x}{2}\right] .
$$

\section{Numerical Tests}

\subsection{Linear Alfvén waves}

We present here the results of the propagation of a sinusoidal linear Alfvén wave as an example. We use 400 grid points, and the grid size $\Delta x$ is taken to be 1 . Periodic boundary conditions are used. As an initial condition, we set $\rho=1, p=0.15, v_{x}=0, \gamma=5 / 3, B_{x}=1$, $B_{y}=\delta B \cos (k x)$, and $v_{y}=-B_{y} / \sqrt{\rho}$, with $k=2 \pi m / L$ where $L, m$, and $\delta B$ represent the system length, mode number, and magnetic field amplitude of Alfvén waves, respectively. We set $m=2$ and $\delta B=0.001 B_{x}$. The time step interval $\Delta t$ is decided so that a CFL number is equal to 0.2 .

Figure 4 shows the profile of $B_{y}$ at $t=10 T$ where $T$ represents a traveling time over a system length at an Alfvén speed. We show results of the present scheme with both van Leer interpolation and non-oscillatory interpolation for the MOC, referring to the van Leer interpolation and the non-oscillatory interpolation as "Scheme A" and "Scheme B", respectively. For comparison, we also show results of the CIP-MOCCT scheme (Kudoh and Shibata, 1997), the second-order Roe-type MUSCL (Monotone Upstreamcentered Scheme for Conservation Laws) scheme (Tanaka, 1994; Fukuda and Hanawa, 1999), and the second-order HLLD-MUSCL scheme (Miyoshi and Kusano, 2005).

The results of Scheme A and the CIP-MOCCT scheme show that the profiles tend to be flat-top and flat-bottom as a wave propagates. This shape is not observed with the non-oscillatory scheme nor the CIP scheme in the advection phase but by the van Leer interpolation which maintains monotonicity of a profile when applying the MOC (see Eq. (17)). Scheme B shows no distortion in the profile in comparison with Scheme A. The reason for this is that the non-oscillatory interpolation in the MOC is reduced to a simple third-order Lagrangian interpolation for a smooth profile, such as sinusoidal waves, and extrema of the profile are adequately captured.

On the other hand, the results with the Roe-MUSCL and the HLLD-MUSCL schemes show a considerable distortion of the profile, especially near extrema, because the minmod limiter (e.g., Hirsch, 1990) used in the MUSCL scheme makes a profile rectangular. 

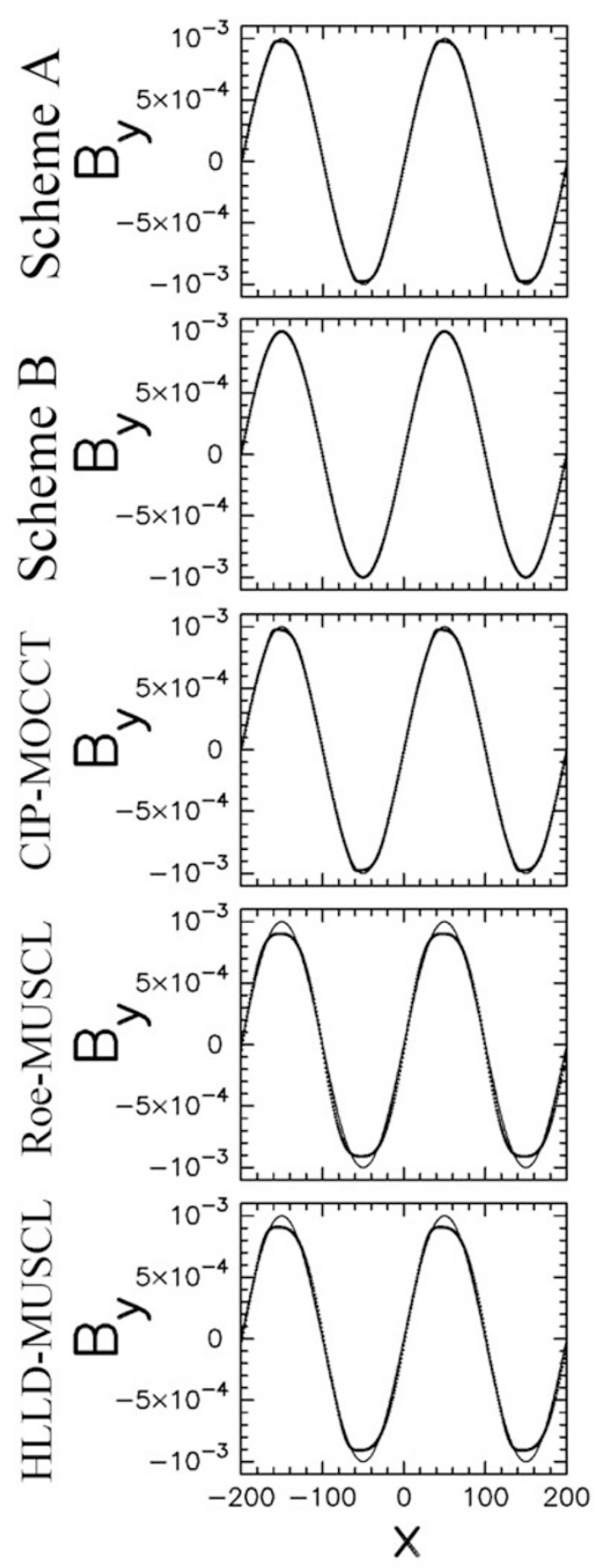

Fig. 4. The propagation of a sinusoidal linear Alfvén wave solved with Scheme A, Scheme B, CIP-MOCCT scheme, second-order Roe-type MUSCL scheme and second-order HLLD-MUSCL scheme. The $y$ component of magnetic field $B_{y}$ is shown. The dotted line and solid line denote a numerical result and an exact solution, respectively.

\subsection{MHD shock tube}

The results of MHD shock tube problems are presented (Brio and $\mathrm{Wu}, 1988$ ). We use 400 grid points, and the grid size $\Delta x$ is taken to be 1 . The ratio of specific heats $\gamma$ is set to be $5 / 3$. As an initial condition, we set $\rho=1.0, p=1.0$, $B_{y}=1, B_{x}=0.75$ for $x<0$, and $\rho=0.125, p=0.1$, $B_{y}=-1, B_{x}=0.75$ for $x>0$. The time step interval $\Delta t$ is chosen so that a CFL number is equal to 0.2 . The profiles of $\rho, v_{x}, v_{y}$, and $B_{y}$ at $t=40$ are shown in Fig. 5. The results of several schemes are shown which are the same as those for tests of sinusoidal Alfvén waves.

We found that the fast rarefaction waves (FR), slow compound wave (SM), contact discontinuity (CD), and slow shock (SS) are well resolved with Scheme A. This result shows good quantitative agreement with those of other schemes (Brio and Wu, 1988; Dai and Woodward, 1994).

The result with Scheme A is generally similar to that observed with the CIP-MOCCT scheme in the following aspects. We observed a small distortion in the velocity and magnetic field profile at the forward propagating fast rarefaction wave. At the contact discontinuity, the density profile also has overshoot and undershoot. These aspects imply that the overshoot and undershoot are due to the third-order interpolant used in the non-oscillatory and the CIP schemes. Scheme A requires a few more grid points than the CIPMOCCT scheme to capture the contact discontinuity. It is noted that the present scheme does not use any additional numerical diffusion term in basic equations, unlike the CIPMOCCT scheme.

On the other hand, Scheme B shows overshoot at the slow shock and considerable oscillations between the slow shock and the fast rarefaction waves. We found that these oscillations were generated at the slow shock and that they propagated to the right. Scheme B needs more improvement to solve discontinuities although this scheme can produce accurate solutions for continuous Alfvén waves, as shown in the previous section.

In contrast, the Roe-MUSCL and the HLLD-MUSCL schemes give better results without overshoot and undershoot although the contact discontinuity somewhat diffuses, primarily because the TVD schemes are well-designed for capturing shocks and discontinuities by enforcing monotonicity. It should be noted that the numerical procedure of the HLLD-MUSCL scheme is much simpler than that of the Roe-MUSCL scheme (Miyoshi and Kusano, 2005).

\section{Summary and Discussion}

We have presented a new numerical scheme for solving MHD equations by applying a non-oscillatory and conservative scheme (Umeda, 2008) to the advection terms and pressure gradient terms. To solve the magnetic stress and induction aspects, we used the MOCCT scheme (Hawley and Stone, 1995). In the MOC, we adopted the van Leer interpolation and the non-oscillatory interpolation. We performed test simulations of sinusoidal Alfvén waves and the MHD shock tube to compare our schemes with recent high-resolution schemes, such as the CIP-MOCCT, RoeMUSCL, and HLLD-MUSCL.

The combination of the non-oscillatory scheme for the advection phase and the van Leer interpolation for the nonadvection phase gives small undershoot and overshoot at the slow shock and fast rarefaction waves in the MHD shock tube test. We observed small flattening at the extrema of profiles in the results of sinusoidal Alfvén waves that is caused by the van Leer limiter in the MOC. The results of the CIP-MOCCT scheme are generally similar to those of the present scheme.

The numerical procedures of these two schemes are similar in which the advection phase and non-advection phase are separately solved with the MOCCT scheme. However, 


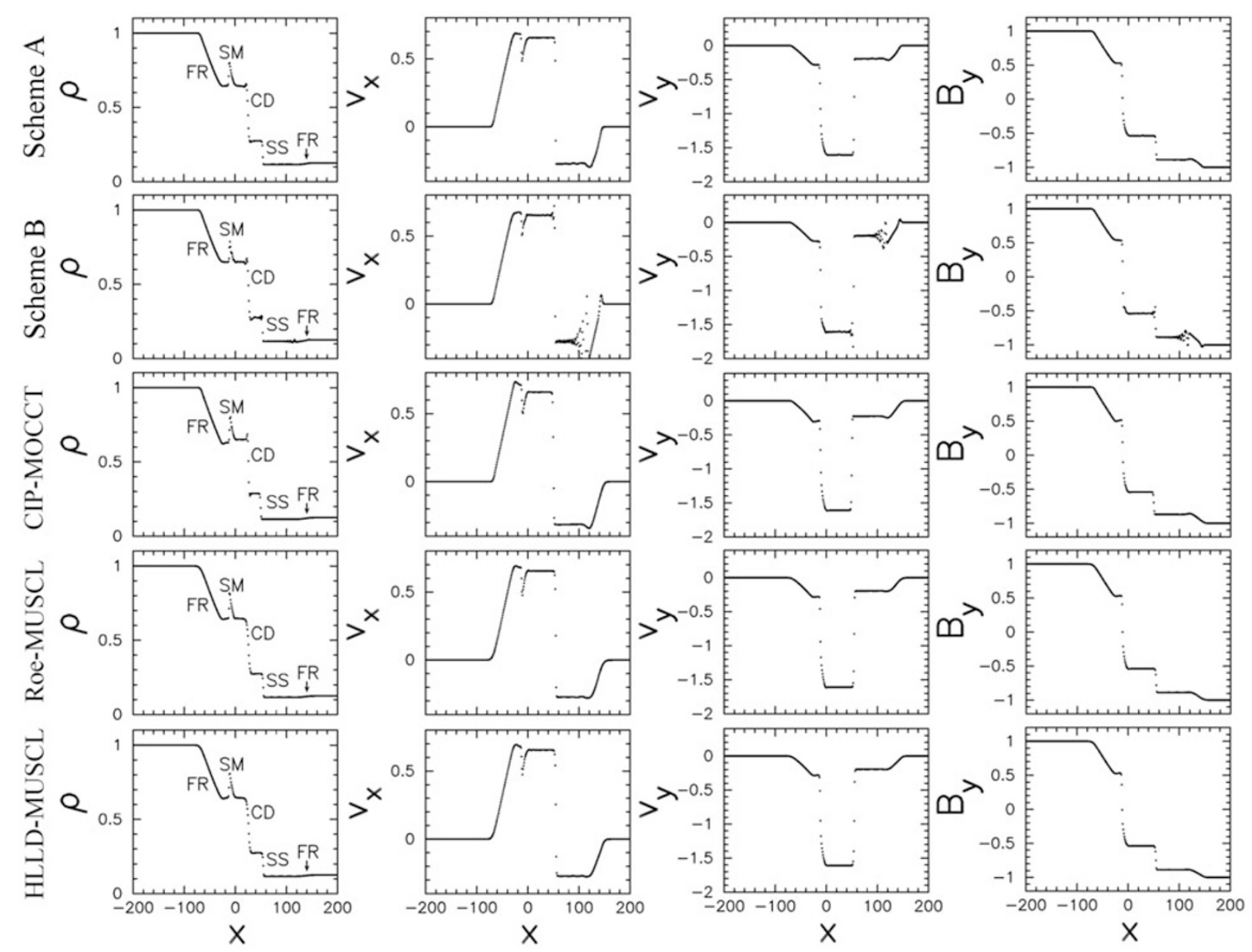

Fig. 5. Numerical solutions to the MHD shock tube problem solved with Scheme A, Scheme B, the CIP-MOCCT scheme, second-order Roe-type MUSCL scheme, and second-order HLLD-MUSCL scheme. The density $\rho, x$ component of velocity $v_{x}, y$ component of velocity $v_{y}$, and $y$ component of magnetic field $B_{y}$ are shown.

in comparison with the CIP-MOCCT scheme, the present scheme has a number of advantages in that it (1) suppresses numerical oscillations without an additional diffusion term; (2) solves the MHD equations in the conservative form; (3) preserves positivity for the density; (4) does not need additional computer memory to store partial derivatives or integrals of the profile. Note that the CIP-type scheme can suppress numerical oscillations by using a rational interpolant instead of a cubic polynomial interpolant (rationalCIP) (Xiao et al., 1996). However, the rational-CIP scheme is not conservative nor necessarily non-oscillatory in twoand three-dimensional systems. Although there are also conservative CIP schemes (Yabe et al., 2001), these require much more computer memory than our scheme, especially in two and three dimensions.

The TVD schemes (Roe-MUSCL and HLLD-MUSCL schemes) can suppress numerical oscillations by automatically controlling a numerical diffusion. However, the results of the sinusoidal wave propagation shows that the TVD scheme tends to make considerable distortions near extrema of the profile due to the MUSCL scheme, while the present scheme and CIP-MOCCT scheme give only small distortions, as shown in Fig. 4. For profiles with extrema, such as sinusoidal waves, the TVD schemes need many more grid points than the present scheme and CIP-MOCCT scheme.

We also attempted to apply the non-oscillatory interpolation to the MOC as an alternative to the van Leer interpolation (Scheme B). The non-oscillatory interpolation gives an accurate solution for sinusoidal Alfvén waves. However, significant oscillations are observed at the slow shock in the MHD shock tube test. To follow discontinuous transverse waves, further improvement is needed to implement the non-oscillatory interpolation into the MOCCT scheme.

The numerical results suggest that numerical oscillations arise from the non-advection phase. Although the nonoscillatory scheme can suppress the generation of spurious extrema in space, the simple Euler time-integration method used in Eqs. (21), (22), and (24) occasionally overestimates the temporal variation of physical quantities per unit time step. The TVD schemes (Roe-MUSCL and HLLD-MUSCL schemes) commonly use a higher-order Runge-Kutta time-integration method. To check the effect of higher-order time-integration methods, the secondorder (two-step) Runge-Kutta time-integration method and the third-order (three-step) Runge-Kutta time-integration method are implemented into Scheme B. The results are shown in the middle and bottom panels of Fig. 6. The result with the first-order time-integration (Euler) method is also shown in the top panel of Fig. 6 as a reference. We con- 

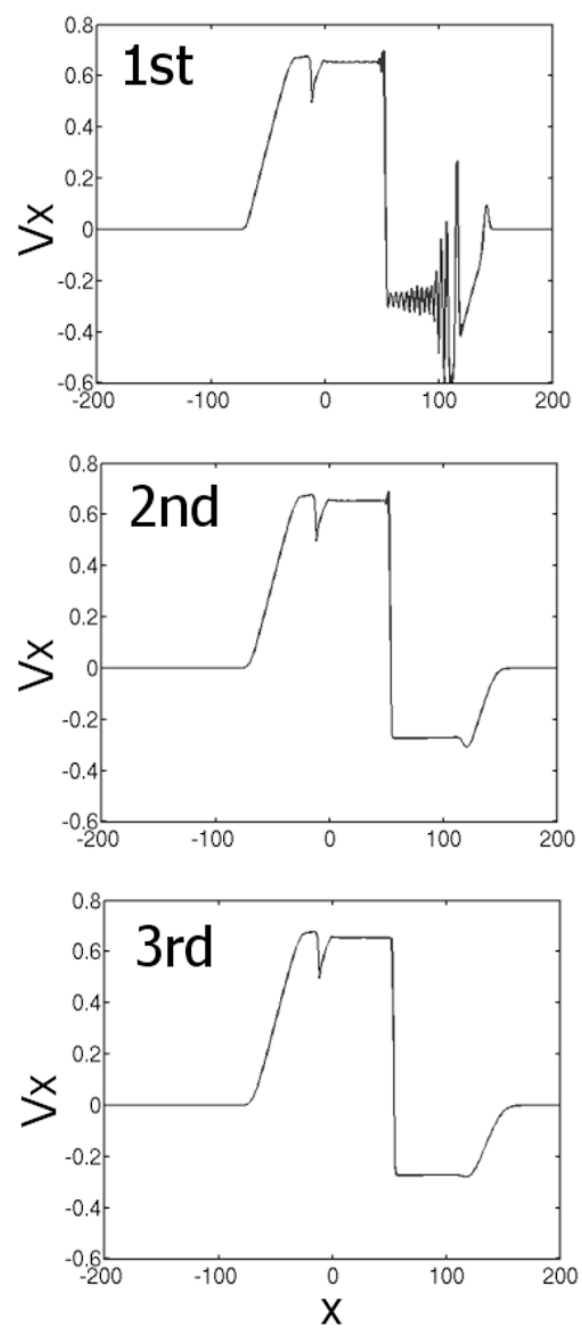

Fig. 6. Numerical solutions to the MHD shock tube problem solved with a combination of Scheme B and first-order time-integration (Euler) method, second-order (two-step) Runge-Kutta time-integration method, or third-order (three-step) Runge-Kutta time-integration method. The $x$ component of velocity $v_{x}$ is shown.

firmed that the use of higher-order time-integration methods can suppress the generation of spurious oscillations arising from the non-advection phase. It should be noted, however, that profiles become rather diffusive with higherorder time-integration methods and that higher-order timeintegration methods require more CPU time. Another reason explaining the presence of numerical oscillations might be the electro-motive force in the CT scheme. In the present method, we do not apply the non-oscillatory interpolation to the induction electric field used in Eq. (33). The centered difference of the induction electric field used in Eq. (33) would be another cause of numerical oscillations.

In summary, we implemented a new non-oscillatory, positivity preserving, and conservative scheme into the MHD equations. We adopted the MOCCT scheme to solve the induction equation and magnetic stress. The implementation of the non-oscillatory scheme and MOCCT scheme enables us to follow the long-term evolution of both Alfvén waves and compressive shocks. To avoid numerical oscillations in the discontinuous Alfvén waves, van Leer's limiter is essen- tial for the MOC.

The scheme presented here has been used for a numerical modeling of Alfvén waves in the solar wind (Tanaka et al., 2007). Monochromatic and circularly polarized Alfvén waves injected from the lower corona are subject to the parametric decay. The sinusoidal Alfvén waves propagating in radially expanding plasmas excite compressive sound waves that steepen into shocks. An accurate solution for the propagation of sinusoidal linear Alfvén waves is needed to obtain the exact growth rate of the parametric decay. The compressive fluctuation also leads to a negative density due to a numerical effect. Thus, it is important to treat both Alfvén waves and shocks. In the present scheme, the MOC follows the evolution of Alfvén waves, while the nonoscillatory scheme suppresses numerical oscillations and preserves density positivity.

Extension of the present scheme to multi-dimensions is left as a future research objective. The non-oscillatory scheme can be applied to advection phases and pressure gradient terms. This approach, which has a simple directional splitting scheme, enables us to obtain reasonable results for numerical tests of multi-dimensional hydrodynamic equations. For the MHD equations, the MOCCT scheme has been extended to multi-dimensions in several schemes (Hawley and Stone, 1995; Kudoh et al., 1998, 1999; Ogata and Yabe, 2004; Ogata et al., 2004). Note that these schemes solve a pressure equation that does not need the MOCCT scheme. In contrast, we solve the energy equation, which does need the MOCCT scheme. Unlike the pressure equation, the energy equation needs timeadvanced velocity and magnetic field, both of which are computed with the MOCCT scheme, as shown in Eq. (22). Thus, we need to develop a technique to solve the energy equation for multi-dimensions.

Distortions of profiles due to the van Leer limiter also need to be resolved. Since the van Leer limiter is essential to solve discontinuous Alfvén waves in the MOC, an alternative method to the MOC may be needed. The nonoscillatory scheme would be implemented into the Riemann solvers, such as the HLLD scheme, as an alternative scheme to TVD schemes.

Acknowledgments. This work was supported by Grant-in-Aid for Young Scientists (Start-up) \#19840024 (T.U.) from JSPS, the 21st Century COE Program "Dynamics of the Sun-EarthLife Interactive System (SELIS)" at Nagoya University (S.T. and Y.M.), and in part Grant-in-Aid for Creative Scientific Research \#17GS0208 "The Basic Study of Space Weather Prediction" from the MEXT of Japan. The MHD codes with the CIP-MOCCT scheme and the second-order Roe-MUSCL scheme are provided by Coordinated Astronomical Numerical Software (CANS), ACTJST. This work was also carried out as a collaborative computational research project at Solar-Terrestrial Environment Laboratory, Nagoya University.

\section{References}

Brio, M. and C. C. Wu, An upwind differencing scheme for the equations of ideal magnetohydrodynamics, J. Comput. Phys., 75, 400-422, 1988.

Dai, W. and P. R. Woodward, Extension of the Piecewise Parabolic Method to multidimensional ideal magnetohydrodynamics, J. Comput. Phys., 115, 485-514, 1994.

Fukuda, N. and T. Hanawa, Gravitational and parametric instabilities of the interstellar medium in which the Alfvén wave travels, Astrophys. J., 
517, 226-241, 1999.

Hawley, J. F. and J. M. Stone, MOCCT: A numerical technique for astrophysical MHD, Comput. Phys. Commun., 89, 127-148, 1995.

Hirsch, C., Numerical Computation of Internal and External Flows, vol. 2: Computational Methods for Invisid and Viscous Flows, Wiley, 1990.

Kudoh, T. and K. Shibata, Magnetically driven jets from accretion disks. II. Nonsteady solutions and comparison with steady solutions, Astrophys. $J .$, 476, 632-648, 1997.

Kudoh, T., R. Matsumoto, and K. Shibata, Magnetically driven jets from accretion disks. III. 2.5-dimensional nonsteady simulations for thick disk case, Astrophys. J., 508, 186-199, 1998.

Kudoh, T., R. Matsumoto, and K. Shibata, Numerical MHD simulation of astrophysical problems by using CIP-MOCCT method, Comput. Fluid Dyn. J., 8, 56-68, 1999.

Miyoshi, T. and K. Kusano, A multi-state HLL approximate Riemann solver for ideal magnetohydrodynamics, J. Comput. Phys., 208, 315344, 2005.

Ogata, Y. and T. Yabe, Multi-dimensional semi-Lagrangian characteristic approach to the shallow water equations by the CIP method, Int. J. Comput. Eng. Sci., 5, 699-730, 2004.

Ogata, Y., T. Yabe, K. Shibata, and T. Kudoh, Efficient computation of magneto-hydrodynamic phenomena in astrophysics by CCUP-MOCCT method, Int. J. Comput. Method, 1, 201-225, 2004.

Roe, P. L., Approximate Riemann solvers, parameter vectors, and difference schemes, J. Comput. Phys., 43, 357-372, 1981.

Ryu, D. and T. W. Jones, Numerical magetohydrodynamics in astrophysics: Algorithm and tests for one-dimensional flow, Astrophys. J., 442, 228-258, 1995.
Tanaka, S., T. Ogino, and T. Umeda, Parametric decay of circularly polarized Alfven waves in the radially expanding solar wind, J. Geophys. Res., 112, A10110, doi:10.1029/2007JA012513, 2007.

Tanaka, T., Finite volume TVD scheme on an unstructured grid system for three-dimensional MHD simulation of inhomogeneous systems including strong background potential fields, J. Comput. Phys., 111, 381-390, 1994.

Umeda, T., A conservative and non-oscillatory scheme for Vlasov code simulations, Earth Planets Space, 60, 773-779, 2008.

Umeda, T., M. Ashour-Abdalla, and T. Schriver, Comparison of numerical interpolation schemes for one-dimensional electrostatic Vlasov code, $J$. Plasma Phys., 72, 1057-1060, 2006.

van Leer, B., Towards the ultimate conservative difference scheme. II. Monotonicity and conservation combined in a second-order scheme, $J$. Comput. Phys., 14, 361-370, 1974.

van Leer, B., Towards the ultimate conservative difference scheme: IV. A new approach to numerical convection, J. Comput. Phys., 23, 276-299, 1977.

Xiao, F., T. Yabe, and T. Ito, Constructing oscillation preventing scheme for advection equation by rational function, Comput. Phys. Commun., 93, 1-12, 1996.

Yabe, T., F. Xiao, and T. Utsumi, The Constrained interpolation profile method for multiphase analysis, J. Comput. Phys., 169, 556-593, 2001.

S. Tanaka, T. Umeda (e-mail: umeda@stelab.nagoya-u.ac.jp), Y. Matsumoto, T. Miyoshi, and T. Ogino 